\title{
Thermal boundary conductance across rough interfaces probed by molecular dynamics
}

\author{
Samy Merabia* \\ Institut Lumière Matière, UMR5306 Université Lyon 1-CNRS, Université de Lyon, F-69622 Villeurbanne cedex, France
}

Konstantinos Termentzidis

LEMTA CNRS-UMR7563, Université de Lorraine, F-54506 Vandoeuvre les Nancy, France

(Received 14 March 2013; revised manuscript received 29 January 2014; published 25 February 2014)

\begin{abstract}
We report on the influence of the interfacial roughness on the thermal boundary conductance between two solids, using molecular dynamics. We show evidence of a transition between two regimes, depending on the interfacial roughness: When the roughness is small, the boundary conductance is constant, taking values close to the conductance of the corresponding planar interface. When the roughness is larger, the conductance becomes larger than the planar interface conductance and the relative increase is found to be close to the increase of the interfacial area. The cross-plane conductivity of a superlattice with rough interfaces is found to increase in a comparable amount, suggesting that heat transport in superlattices is mainly controlled by the boundary conductance. These observations are interpreted using the wave characteristics of the energy carriers. We characterize also the effect of the angle of the asperities and find that the boundary conductance displayed by interfaces having steep slopes may become important if the lateral period characterizing the interfacial profile is large enough. As a result, triangular-shaped interfaces may be used to enhance the conductance of planar interfaces by a factor greater than three. Finally, we consider the effect of the shape of the interfaces and show that the sinusoidal interface displays the highest conductance because of its large true interfacial area. All of these considerations are relevant to the optimization of nanoscale interfacial energy transport.
\end{abstract}

DOI: 10.1103/PhysRevB.89.054309

PACS number(s): 68.35.Ja, 07.05.Tp, 44.10.+i

\section{INTRODUCTION}

The existence of a finite thermal boundary resistance between two solids has important practical consequences, especially in the transport properties of nanostructured materials. When the distance between interfaces becomes submicronic, heat transfer is mainly controlled by the interfacial phonon transmission, which in turn governs the thermal boundary resistance. In certain applications, such as electro-optical modulators [1], optical switching devices [2], and pressure sensors [3], a low resistance is desired to enhance energy flow. In thermoelectric devices, on the contrary, a large resistance is preferable so as to generate large barriers for a wide class of phonons [4-6]. Two strategies may be followed in order to tune the value of the boundary resistance between two solids. Either the solid/solid interaction is changed through the coupling with a third body, which is usually a self-assembled monolayer $[7,8]$, or the other possibility is to modulate the interfacial roughness [9]. This latter direction has been illustrated experimentally through chemical etching [10-12]. However, a theoretical model describing the effect of the interfacial roughness on the thermal boundary conductance at room temperature is still lacking $[13,14]$. Note that the role of the interfacial roughness on the Kapitza conductance was identified a long time ago, in the context of liquid helium/metal interfaces at very low cryogenic temperatures [15,16]. At these temperatures, the phonon coherence length may be comparable with the typical heights of the interface, leading to strong phonon scattering which is put forward to explain the high values of the conductance experimentally reported, as compared with the classical acoustic mismatch theory which

\footnotetext{
*samy.merabia@univ-lyon1.fr
}

assumes planar interfaces [17]. Such considerations have received less attention for room-temperature solids, probably because in this case the phonon coherence length is very small.

Understanding the role of the interfacial roughness also has important consequences in the transport properties of superlattices, which are good candidates for thermoelectric conversion materials, thanks to their low thermal conductivity [18]. Designing superlattices with rough interfaces has been recently achieved, opening an avenue for reducing the thermal conductivity in the direction perpendicular to the interfaces [19]. Again, the physical mechanisms at play in the heattransport properties of rough superlattices have not been elucidated so far. Molecular dynamics offers a privileged route to understand the interaction between the energy carriers in a solid and the asperities of the interface [20-22]. In this paper, we use molecular dynamics to probe interfacial heat transfer across a model of rough interfaces. Because of the difficulty to determine the temperature jump across a nonplanar interface, we have used thermal-relaxation simulations, which enable one to compute the thermal boundary resistance characterizing rough interfaces. In Sec. II, we describe the structures used to probe the conductance of rough interfaces. In Sec. III, we explain the methodology retained to extract the thermal boundary conductance from molecular dynamics. The simulation results are presented and discussed in Sec. IV. We first concentrate on model interfaces made of isosceles triangles. For these model interfaces, we present the results for the thermal conductance as a function of the interfacial roughness and interpret the results using a simple acoustic model in Sec. IV B. In the following section, we characterize the effect of the angles of the asperities. Finally, in Sec. IV D, we have appraised the effect of the interfacial shape. We discuss the consequences of this work in Sec. V. 


\section{STRUCTURES AND SAMPLE PREPARATION}

We will consider a model of rough interfaces, constructed from two perfect fcc Lennard-Jones solids whose interface is orientated along the crystallographic [100] direction, as represented in Fig. 1. We introduce some two-dimensional (2D) roughness in the $x z$ plane, where $x$ and $z$ denote, respectively, the [100] and [001] directions. As we use periodic boundary conditions in all spatial directions, the system studied is similar to a superlattice. The dimension in the $y$ direction has been fixed to $10 a_{0}$, where $a_{0}$ is the lattice parameter, while the dimension in the $z$ direction- the superlattice period-has been varied between 5 and $40 a_{0}$. All of the atoms of the system interact through a Lennard-Jones (LJ) potential, $V_{\mathrm{LJ}}(r)=4 \epsilon\left[(\sigma / r)^{12}-(\sigma / r)^{6}\right]$, truncated at a distance $2.5 \sigma$. A single set of energy $\epsilon$ and diameter $\sigma$ characterizes the interatomic interaction potential. As a result, the two solids have the same lattice constant $a_{0}$. To introduce an acoustic mismatch between the two solids, we have considered a difference between the masses of the atoms of the two solids, characterized by the mass ratio $m_{r}=m_{2} / m_{1}$. In all of the following, we will use $m_{r}=2$, which has been shown to give an impedance ratio typical of the interface between $\mathrm{Si}$ and $\mathrm{Ge}$ [22]. From now on, we will use real

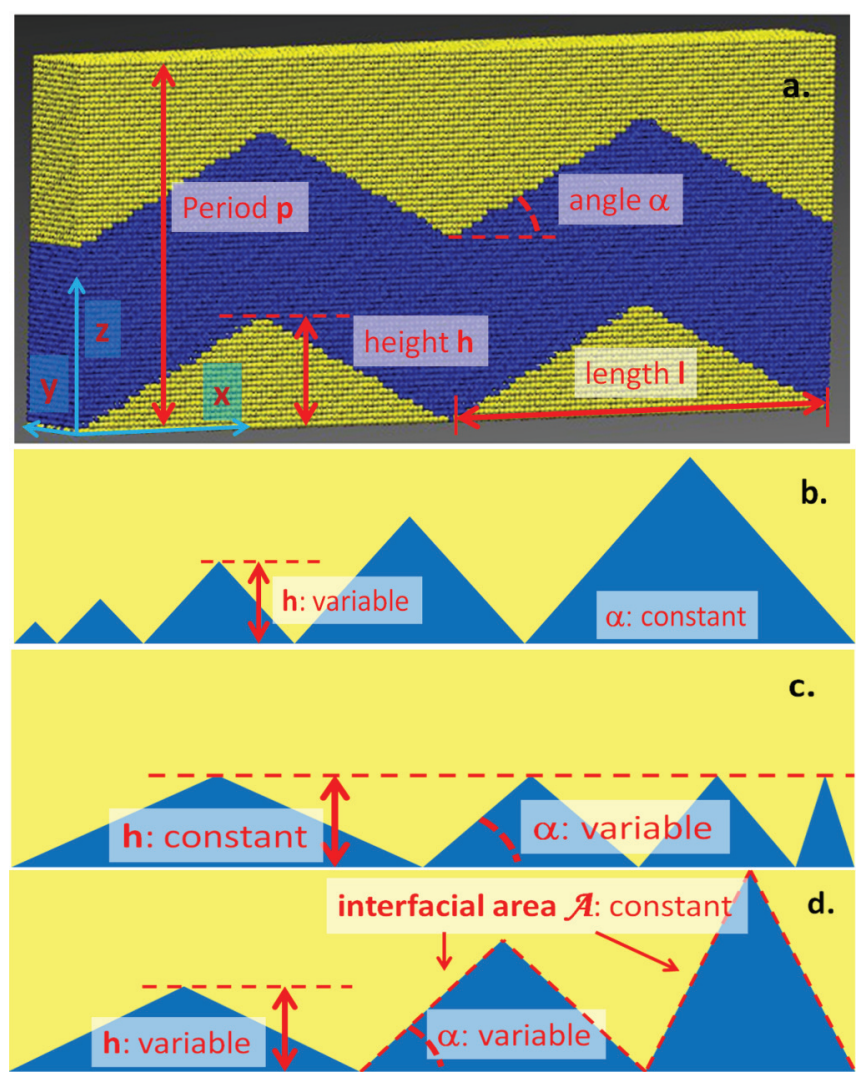

FIG. 1. (Color online) (a) Diagram showing the different parameters used as nomenclature for the triangular-shaped interfaces. (b)-(d) Schematic representation of the different parameters that have been tested: (b) interfacial height at a constant value of the angle $\alpha=45 \mathrm{deg}$, (c) angle $\alpha$ at a constant value of the interface height $h$, and (d) angle $\alpha$ and interfacial height $h$ at a constant value of the interfacial area $\mathcal{A}$. units where $\epsilon=1.67 \times 10^{-21} \mathrm{~J} ; \sigma=3.4 \times 10^{-10} \mathrm{~m}$, and $m_{1}=6.63 \times 10^{-26} \mathrm{~kg}$, where these different values have been chosen to represent solid argon. With this choice of units, the unit of time is $\tau=\sqrt{m \sigma^{2} / \epsilon}=2.14 \mathrm{ps}$ and the unit of interfacial conductance is $G=k_{B} /\left(\tau \sigma^{2}\right) \simeq 56 \mathrm{MW} / \mathrm{K} / \mathrm{m}^{2}$. The different interfaces have been prepared as follows: First the structures have been generated by mapping the space with fcc structures using the lattice parameter of the fcc LJ solid at zero temperature [23]: $a_{0}(T=0 K)=1.5496 \sigma$. The structures have been equilibrated at the two final finite temperatures $T=40$ and $T=18 \mathrm{~K}$ using a combination of a Berendsen, a Nosé Hoover thermostat, and a barostat at 0 atm [24]. The total equilibration time lasts one million time steps which corresponds to a total time of $4,28 \mathrm{~ns}$ with a time step $d t=4.28 \mathrm{fs}$. The equilibrium lattice parameters have been found to be $a=1.579 \sigma$ at $T=40 \mathrm{~K}$ and $a=1.5563 \sigma$ at $T=18 \mathrm{~K}$. In this paper, we consider different types of rough interfaces, as represented in Fig. 1. The first one consists of triangular-shaped interfaces having a constant angle $\alpha=45 \mathrm{deg}$ with respect to the $x y$ plane and a variable height $h$ [Fig. 1(b)]. In the second type of interface analyzed, we keep constant the interfacial height $h$ and we vary the angle $\alpha$ [Fig. 1(c)]. In the third case, both the angle and the height are varied, keeping constant the interfacial area $\mathcal{A}$. Finally, the effect of the shape of the interfaces has also been appraised considering totally rough interfaces, small triangles juxtaposed on triangular interfaces, and square- and wavy-shaped interfaces. This analysis covers all of the possible parameters that might be involved in the geometry of the interfaces with the aim to quantify their influence on phonon interfacial transport.

\section{THERMAL BOUNDARY CONDUCTANCE FROM THERMAL-RELAXATION SIMULATIONS}

In this section, we briefly review the methodology adopted to probe the interfacial conductance between two solids, using relaxation simulations. Generally speaking, there are different methods to extract the boundary conductance from molecular simulations: one method consists of applying a net heat flux $q$ across the system through the coupling of two energy reservoirs and measuring the finite-temperature jump $\Delta T$ across the interface [25,26]. This allows one to compute the interfacial conductance $G_{K}=q / \Delta T$. For the rough interfaces that we will consider in the following, it may be difficult to clearly identify a temperature jump, especially if the roughness is large. On the other hand, relaxation simulations do not require one to spatially resolve the temperature field in the vicinity of the interface, and for this reason they are well adapted to the determination of the conductance of imperfect interfaces. The principle is akin to the thermoreflectance technique and consists of instantaneously heating one of the two solids and recording the temporal evolution of the temperature of the hot solid [27-29]. The conductance $G_{K}$ is then obtained from the time $\tau$ characterizing the thermal relaxation of the hot solid,

$$
G_{K}=\frac{3 N_{1} k_{B}}{4 \mathcal{A}_{0} \tau},
$$




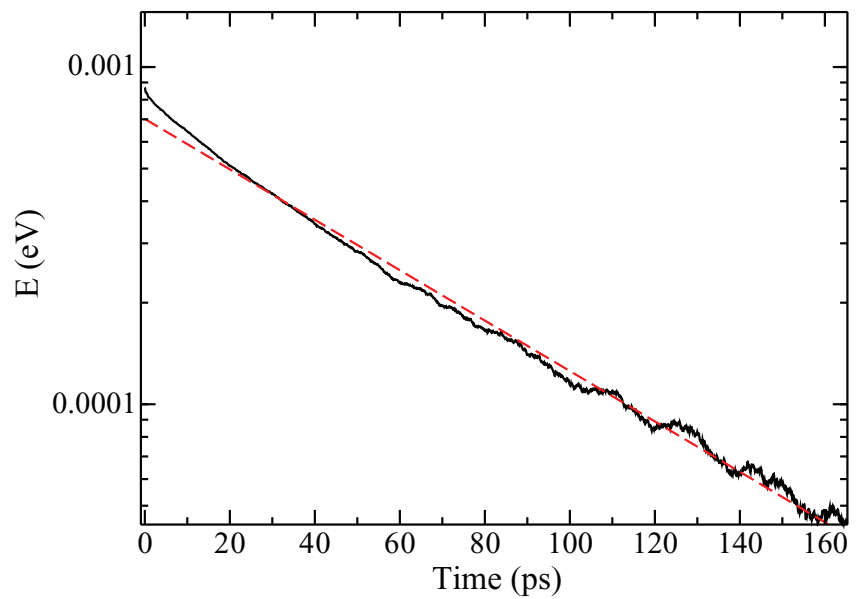

FIG. 2. (Color online) Energy decay of the heated solid obtained using thermal-relaxation simulations. Dashed lines show the exponential fit. The parameters are total length $=40 a_{0}$, temperature $T=40 \mathrm{~K}$, and mass ratio $m_{r}=2$.

where $N_{1}$ is the number of atoms of solid $1, k_{B}$ is the Boltzmann's constant, $\mathcal{A}_{0}$ is the interfacial area projected along the mean normal vector to the interface (corresponding to direction $z$ on Fig. 1), and the factor 4 accounts for the presence of two interfaces. Because the temperature of the heated solid may display some oscillations which may make the determination of the time constant $\tau$ difficult, we have instead used the decay of the energy $E_{1}$ to extract $\tau$,

$$
E_{1}^{m}=\sum_{j \in 1} \frac{1}{2} m \vec{v}_{j}^{2}+\sum_{j, k \in 1} V\left(\vec{r}_{j}-\vec{r}_{k}\right),
$$

where the first term is the kinetic energy and the second term corresponds to the interatomic potential, which here is supposed to be pairwise. An example of the time decay of the energy during thermal relaxation of the hot solid is displayed in Fig. 2, showing that the exponential decay hypothesis is reasonable.

In practice, after equilibration of the system, we have heated one of the two solids and followed the thermal relaxation of the system at constant energy and volume. The heating is performed by instantaneously rescaling the velocities of the hot solid by the same factor. In this paper, we have used a constant value of the temperature increment of $\Delta T=18 \mathrm{~K}$, and checked also that using a smaller value $(\Delta T=8 \mathrm{~K})$ does not significantly change the measured conductances. To remove any contribution stemming from internal phonon scattering in the heated solid, we have run in parallel simulations across the interface between identical solids and calculated the corresponding internal resistance. The Kapitza conductance calculated in this paper has been obtained after having subtracted this internal resistance,

$$
1 / G_{K}=1 / G_{12}-1 / G_{11}
$$

where $G_{12}$ is the conductance measured for the interface between solid 1 and solid 2 , and $G_{11}$ is the conductance measured between identical solids using Eq. (1). This latter conductance is typically twice as large as $G_{12}$ : for a flat interface equilibrated at $T=18 \mathrm{~K}$, one measures
$G_{12}=143 \mathrm{MW} / \mathrm{m}^{2} / \mathrm{K}$ and $G_{11}=336 \mathrm{MW} / \mathrm{m}^{2} / \mathrm{K}$ resulting in a conductance $G_{K} \simeq 248 \mathrm{MW} / \mathrm{m}^{2} / \mathrm{K}$. Note also that Eq. (3) assumes that internal phonon-phonon scattering in the hot solid is the same in the interfacial and in the no-interface system, an assumption which should be verified in the limit of thick solid media. The procedure described above has been followed for all of the systems studied in this paper. Finally, for the simulations discussed in this paper, we have used between five and ten independent configurations, depending on the system size, to determine the value of the Kapitza conductance and the error bar has been found to be typically $15 \%$.

\section{RESULTS}

In this section, we present the simulation results obtained using the relaxation simulations, as detailed above. We will successively study the effect of the interfacial roughness, the angle of the asperities, and the shape of the interface. A summary of the different parameters that will be varied is depicted in Fig. 1.

\section{A. Effect of the superlattice period and number of periods}

In this section, we first quantify finite-size effects in the determination of the conductance of rough interfaces, as measured by Eq. (1). It is important to note that the system simulated is not a single isolated interface, but rather a superlattice because of the periodic boundary conditions. It is thus relevant to assess the influence of the superlattice period on the thermal conductance as measured by Eq. (1). To this end, we will consider a model of rough interfaces, made of isosceles triangles, as depicted in Fig. 1. In Fig. 3, we report the conductance of triangular-shaped interfaces having a fixed roughness height of 32 monolayers (MLs) and a varying period. For the two temperatures considered, the thermal conductance is found to decrease with the system size for short periods, and then saturates for periods larger than $30 \mathrm{~nm}$. The increase of the conductance for thin layers may

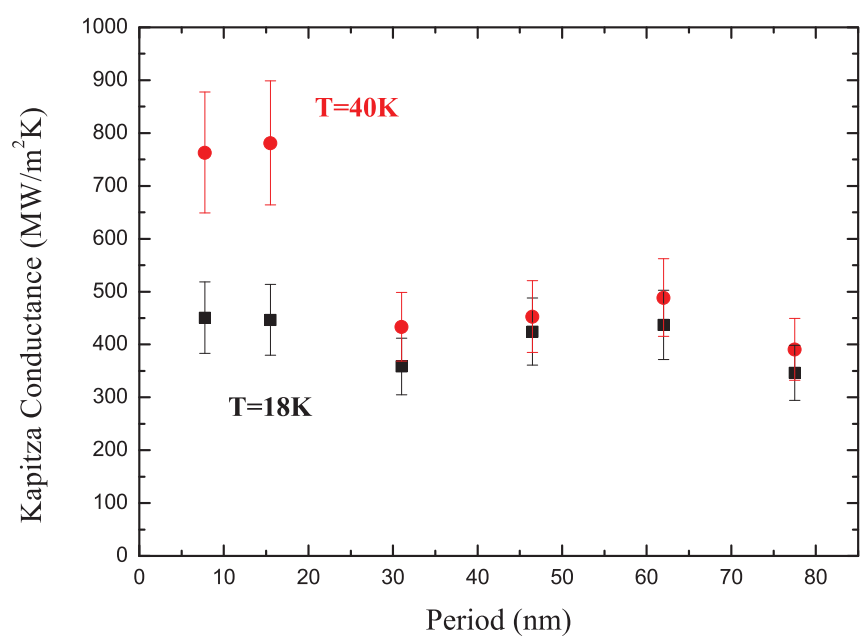

FIG. 3. (Color online) Thermal boundary conductance of isosceles-triangular-shaped interfaces having a roughness height $h=32$ MLs, as a function of the period $p$ defined in Fig. 1. Top: $T=40 \mathrm{~K}$; bottom: $T=18 \mathrm{~K}$. The mass ratio is $m_{r}=2$. 


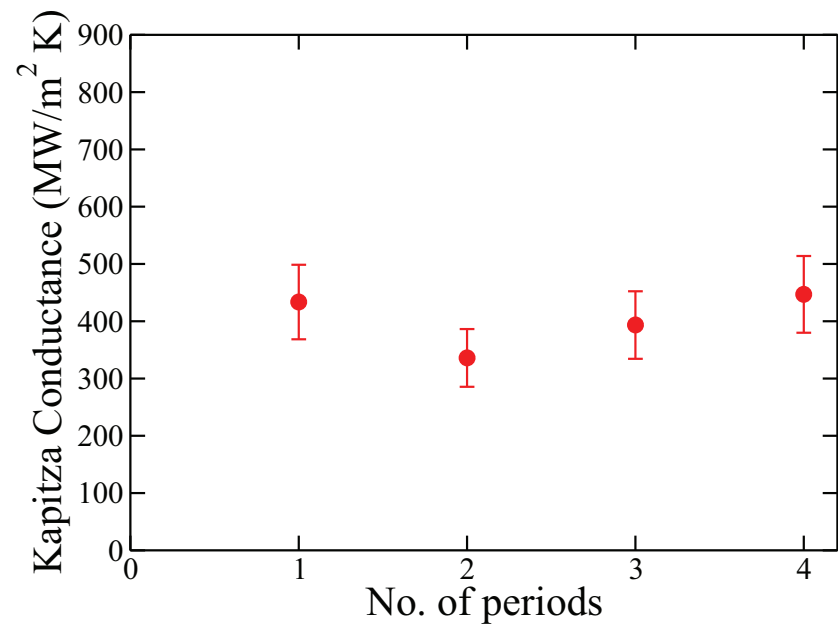

FIG. 4. (Color online) Thermal boundary conductance of isosceles-triangular-shaped interfaces having a roughness height $h=32$ MLs and a period $40 a_{0}$, as a function of the No. periods of the superlattice. The temperature is $T=40 \mathrm{~K}$ and the mass ratio is $m_{r}=2$.

be explained because long wavelength phonons will not see two independent interfaces, but rather a single one. A similar trend has also been reported in lattice dynamics simulations [30] and Green's-function calculations [31]. For thick layers, the conductance measured is constant and has converged to the value characterizing an infinitely thick film. We remark also that the conductance is higher at high temperatures. Generally speaking, the thermal boundary conductance is found to increase with temperature, a trend often attributed to the existence of inelastic phonon scattering at the interface [25,32]. This behavior is consistent with our simulation data. In the following, we will fix the period to $20 a_{0} \simeq 30 \mathrm{~nm}$ as it leads to moderate finite-size effects as already found for superlattices with planar interfaces [33]. Finally, since the system we simulate is akin to a superlattice because of the periodic boundary conditions, it is important to probe the effect of the number of periods on the measured conductance. Figure 4 quantifies the effect of the number of interfaces on the conductance measured in thermal-relaxation simulations. From this figure, we can conclude that within error bars, the number of interfaces has a mild effect on the conductance that we calculate. This is the behavior expected, as we probe a quantity characterizing the interface solely, independently of the number of interfaces.

\section{B. Effect of the interfacial roughness}

We will now concentrate on the influence of the interfacial roughness on the thermal boundary conductance. We will consider rough interfaces having an angle $\alpha$ fixed at $\alpha=$ $45 \mathrm{deg}$, while the height $h$ of the interface is increased so as to change the interfacial roughness, as seen in Fig. 1(b). For the following, it is important to keep in mind that when varying the height of the interface $h$ at a constant value of the angle, the total interfacial area remains constant and larger by a factor $1 / \cos \alpha=\sqrt{2}$ than its corresponding projection on the horizontal $x y$ plane.
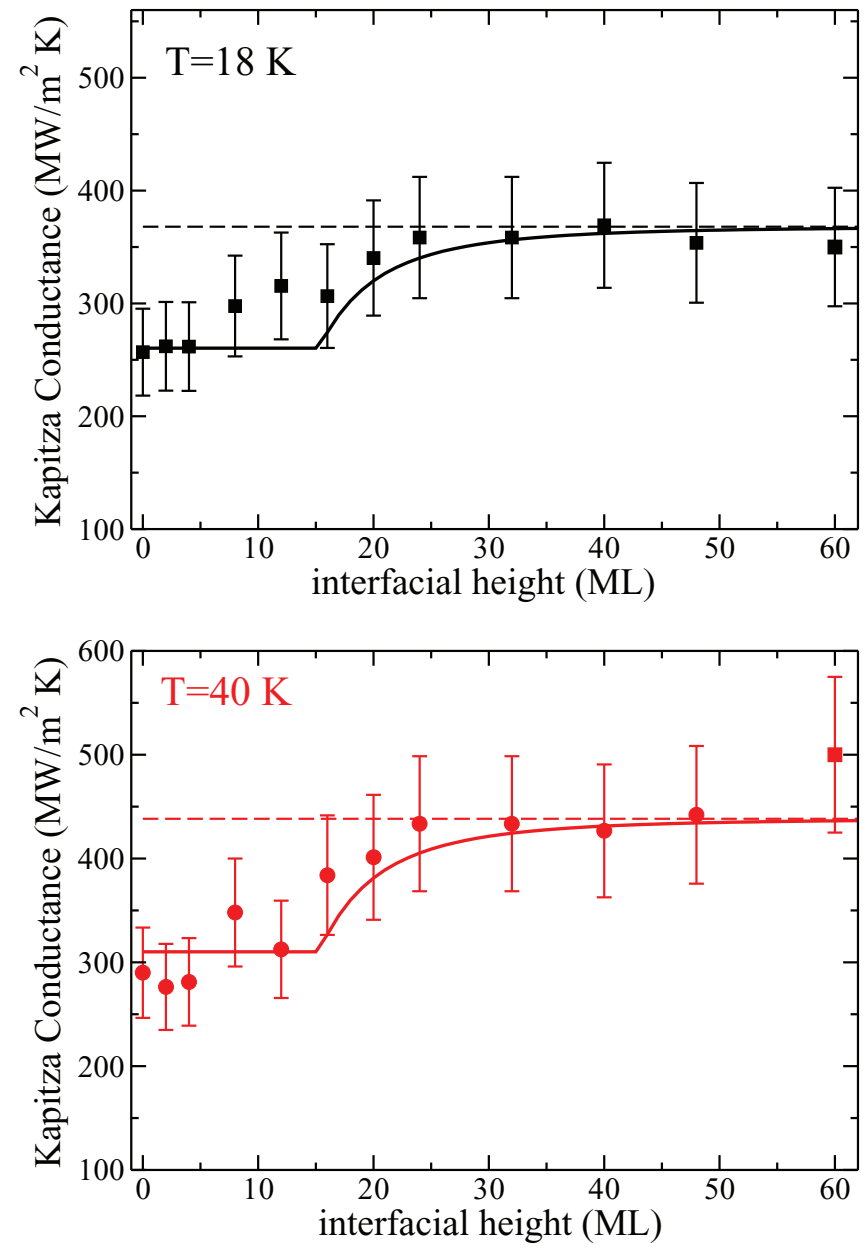

FIG. 5. (Color online) Thermal boundary conductance of isosceles-triangular-shaped interfaces as a function of the interfacial roughness here measured in monolayers $\left(1 \mathrm{ML}=a_{0} / 2 \simeq 0.75 \mathrm{~nm}\right)$. Top: $T=18 \mathrm{~K}$; bottom: $T=40 \mathrm{~K}$. We have also indicated the conductance of the corresponding planar interfaces $(\mathrm{ML}=0)$ and very rough interface $(\mathrm{ML}=60)$. The horizontal dashed lines show the conductance obtained after rescaling the conductance of the planar interface by the true interfacial area. The solid lines denote the theoretical model (5) with the parameter $\xi=0.2$. The parameters are total length $=40 a_{0}$ and mass ratio $m_{r}=2$.

Figure 5 displays the evolution of the measured Kapitza conductance as a function of the interfacial roughness for two temperatures. The conductance of a planar interface, which corresponds to the value $h=0$, has been also indicated for the sake of comparison. Two regimes are to be distinguished, depending on the roughness of the interface $h$. When the height is smaller than typically 20 monolayers (MLs), the conductance seems to be constant or slightly decreases with the roughness, taking values close to the planar interface conductance. When the interfacial height becomes larger, the conductance suddenly increases and tends to saturate for very rough surfaces.

Interestingly, the increase of the conductance between planar and very rough surfaces is found to be close to the increase of the interfacial area. This is materialized in Fig. 5, where we have shown with dashed lines the value of the 


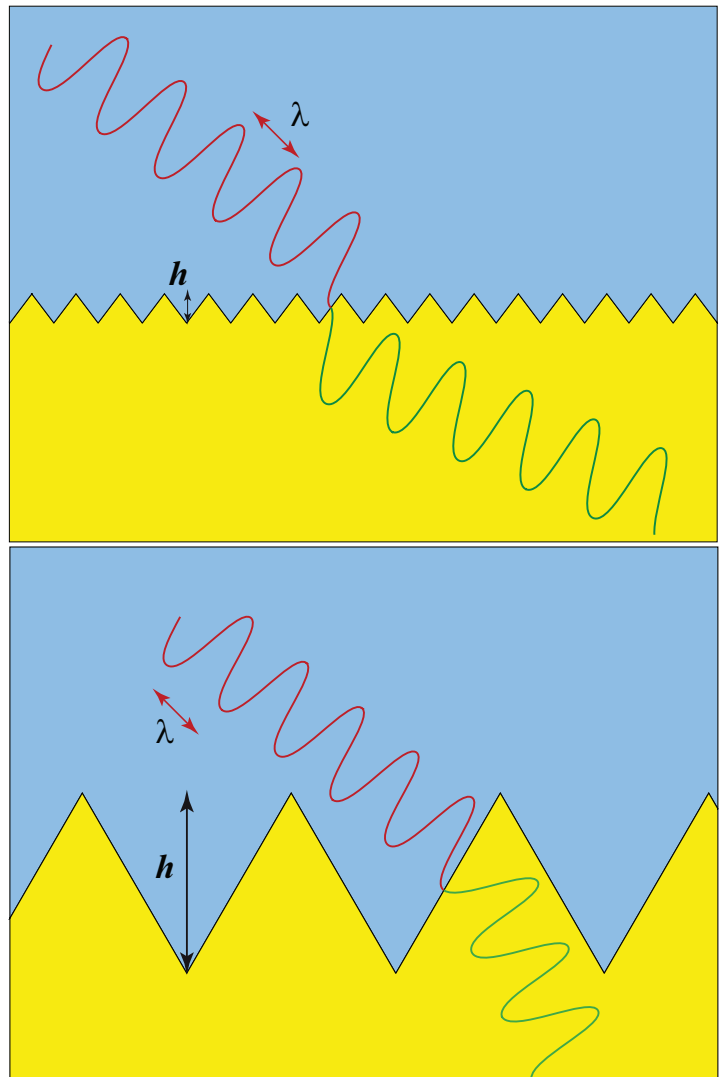

FIG. 6. (Color online) Schematic representation of the roughness-induced phonon scattering. Top: case of a small roughness. The huge majority of incoming phonons see the interface as a plane, and the transmitted heat flux is proportional to the projected interface area. Bottom: case of a large roughness. Most of the phonons have a wavelength larger than the interfacial roughness, and the transmitted heat flux is proportional to the true surface area. For the sake of the representation, we have not drawn the reflected waves. Note also that the phonon wavelength is generally not conserved at the passage of the interface.

conductance obtained by multiplying the conductance of a planar interface by a factor $1 / \cos \alpha$. On the other hand, we have reported in a previous study [20] that the cross-plane thermal conductivity of superlattices with rough interfaces is greater than the conductivity of perfect superlattices by a factor between 1.3 and 1.5 , which encompasses the value $\sqrt{2} \simeq 1.41$. This reinforces the message according to which the thermal conductivity of a superlattice is mainly controlled by the Kapitza resistance exhibited by the interfaces, which in turn seems to be primarily governed by the interfacial area.

We now give some qualitative elements to understand the previous simulation results regarding the influence of the interfacial roughness on the Kapitza conductance. At this point, it is important to have in mind that in the situations that we have modeled, the energy carriers are phonons which are classically populated. A given phonon mode is characterized among others by its wavelength $\lambda$, which may take practically any value between an atomic distance $2 a_{0}$ and the simulation box length $L$ [34]. First, let us concentrate on the case of a small roughness $h$, as shown in Fig. 6 . In this case, the majority of phonon modes have a wavelength larger than $h$ and they see the interface as a planar one: the transmitted heat flux is then controlled by the projected area. On the other hand, when the interface is very rough, most of the phonons have a wavelength smaller than the height $h$, obviously the phonons no longer feel the interface as planar, phonon scattering becomes completely incoherent, and the transmitted heat flux is controlled by the true surface area.

To put these arguments on quantitative grounds, we will consider the following expression of the thermal conductance, inspired by the classical acoustic mismatch model (AMM) model [35]. We introduce a mode-dependent fraction $\psi(\lambda)$, which depends on the considered wavelength and which is equal to 1 when the wavelength is supposed to be small compared with the interfacial roughness $h$, and equal to 0 in the opposite case. We define a dimensionless parameter $\xi$, such that

$$
\begin{aligned}
& \psi(\lambda)=1 \text { if } \lambda<\xi h, \\
& \psi(\lambda)=0 \text { otherwise. }
\end{aligned}
$$

The parameter $\xi$ will be the adjustable fitting parameter of the model. The interfacial conductance is then supposed to be given by

$$
\begin{aligned}
G_{K} & =\frac{3}{2} \zeta \rho k_{B} c_{1}\left\{\int_{0}^{\omega_{\mathrm{Dmin}}} g(\omega) \psi(\lambda) d \omega \mathcal{I}_{12}\right. \\
& \left.+\frac{\mathcal{A}}{\mathcal{A}_{0}} \int_{0}^{\omega_{\mathrm{Dmin}}} g(\omega)[1-\psi(\lambda)] d \omega \mathcal{I}_{12}\right\},
\end{aligned}
$$

where $\rho$ is the crystal number density, $c_{1}$ is the average sound velocity in medium 1 , and $\omega_{\text {Dmin }}$ is the Debye frequency of the softer solid. The parameter $\zeta$ is a scaling factor which accounts for the tendency of the AMM model to overpredict the measured Kapitza conductance [33]. The integral $\mathcal{I}_{12}$ involves the angular-dependent transmission coefficient,

$$
\mathcal{I}_{12}=\int_{0}^{1} \frac{4 Z_{1} \mu_{1} Z_{2} \mu_{2}}{\left(Z_{1} \mu_{1}+Z_{2} \mu_{2}\right)^{2}} \mu_{1} d \mu_{1},
$$

where $Z_{i}=\rho_{i}^{m} c_{i}$ are the acoustic impedances of the two solids, $\rho_{i}^{m}$ is the mass density, and $\mu_{1}=\cos \theta_{1}$ is a shorthand notation to denote the cosine of the phonon incident angle [33]. Finally, the quantity $\mathcal{A} / \mathcal{A}_{0}$ is the ratio of the true interfacial area over the projected one. The physical motivation of Eq. (5) is simple: phonons having a wavelength $\lambda$ larger than $\xi h$ contribute to a transmitted heat flux proportional to the projected area $\mathcal{A}_{0}$, while phonon modes having a wavelength smaller than $\xi h$ contribute to the transmitted heat flux proportionally to the true surface area. We have compared the prediction of Eq. (5) to the simulation results discussed before. To this end, we have assumed Debye solids, with a vibrational density of states $g(\omega)=\omega^{2} /\left(2 \pi^{2} c_{1}^{3}\right)$ and, for the sake of consistency, the mode-dependent wavelength $\lambda$ has been taken to be simply related to the frequency $\omega$ : $\lambda=2 \pi c_{1} / \omega$. Figure 5 compares the predictions of Eq. (5) to the simulations results. The values of the planar interface conductance have been rescaled by a factor $\zeta=3$ and 4 at the temperatures $T=18$ and $T=40 \mathrm{~K}$, respectively. These correction factors account for the fact that the simple AMM model relies on several assumptions, i.e., Debye solids and 
elastic scattering, which may lead to a discrepancy with the molecular dynamics (MD) value. We have chosen the two factors because they yield good agreement with the MD value for smooth interfaces. Apart from this rescaling, the parameter $\xi$ has been treated as the only fitting parameter. Figure 5 shows that a good agreement is found using the value $\xi=0.2$ for the two temperatures considered. The small value of the fitting coefficient may be understood in the following way: Consider a given phonon mode. If its wavelength is larger than the roughness $h$, the effective scattering area would be the projected one. On increasing the roughness, $h$ will become comparable with $\lambda$ and the interface will strongly scatter the considered phonon in all directions. This will contribute a to slight decrease of the conductance, as compared with the planar case, in agreement with the simulation data points. It is only when the roughness becomes very large as compared with the wavelength $\lambda \ll h$ that interfacial scattering becomes again negligible and the transmitted energy is proportional to the true area. The fitting procedure concludes that this regime is reached when the wavelength becomes smaller than typically one-fifth of the interfacial roughness.

\section{Effect of the angle of the asperities}

So far, we have considered the case where the angle $\alpha$ was constant. We now discuss the effect of varying the slope of the model interfaces on the interfacial energy transfer. First, we will change the angle at a fixed value of the interfacial height $h$, as represented in Fig. 1(c). Figure 7 shows the evolution of the Kapitza conductance as a function of the angle, at the two considered temperatures. The constant height $h$ used here corresponds to the regime of large roughnesses in terms of Fig. 5 discussed before. We have also indicated the conductance of a planar interface, for the sake of comparison. The evolution of the conductance with the asperities angle

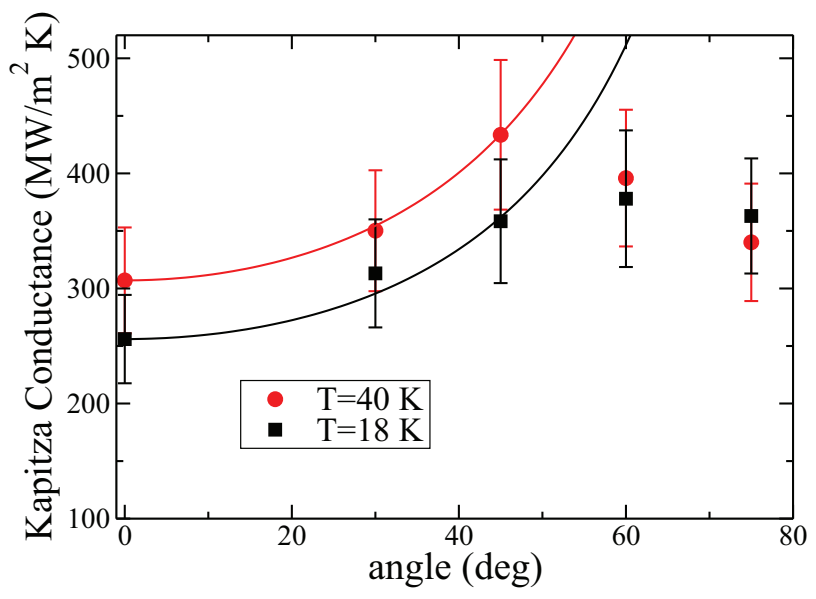

FIG. 7. (Color online) Thermal boundary conductance as a function of the angle of the asperities $\alpha$. The height of the asperities is fixed here and equal to $24 \mathrm{ML}$. The points corresponding to $\alpha=0$ denote the conductance of a planar interface. The solid lines show the interfacial conductance rescaled by the true surface area: $G_{K}=G_{K}(\alpha=0) / \cos \alpha$. The other parameters are total length $=40$ $a_{0}$ and mass ratio $m_{r}=2$. seems to be nonmonotonous: first, it increases for low angles, reaches a maximum for an asperities angle between 30 and 45 degrees, and then decreases when the angle becomes large. In particular, the conductance for an angle greater than $60 \mathrm{deg}$ becomes smaller than the planar interface conductance. This is all the more remarkable as in this latter case, where the true surface area may increase by a factor of four as compared to the planar interface. This discrepancy is best shown after comparing the simulation results with the rescaled conductance $G_{K}(\alpha=0) / \cos \alpha$, which accounts for the increased surface area induced by the asperities. It is immediately clear that for the lowest values of the asperities angles, $\alpha=30$ and $45 \mathrm{deg}$, the rescaled conductance seems to reasonably describe interfacial energy transfer. On the other hand, at large values of $\alpha$, the theoretical expression greatly overestimates interfacial transport. Two phenomena may explain the poor conductance reported: first, on increasing the angle, phonon multiple scattering and backscattering may contribute to diminish interfacial transmission. This has been evidenced by Rajabpour et al. using Monte Carlo ray tracing calculations [21]. Second, for the steep slope interfaces considered here, the effective surface area seems to be the projected one, not the true area, even if the height of the asperities is large. This may be understood qualitatively because for steep interfaces, even if the height is large, the lateral correlation length $l=h / \tan \alpha$ may become comparable with the phonon wavelengths, and the effective interfacial area becomes the projected one. For these steep interfaces, the regime where the transmitted heat flux is controlled by the true surface area should occur at a very large value of the interfacial height $h$. To verify this assessment, we have run simulations where the true surface area has been kept constant [cf. Fig. 1(d)]. The results are displayed in Fig. 8, which concludes a different scenario as compared to the evolution shown previously in Fig. 7. The evolution of the conductance with the angle is no longer nonmonotonous as previously observed, but it rather increases monotonously with $\alpha$. For the relatively small values of the angles $\alpha$, the

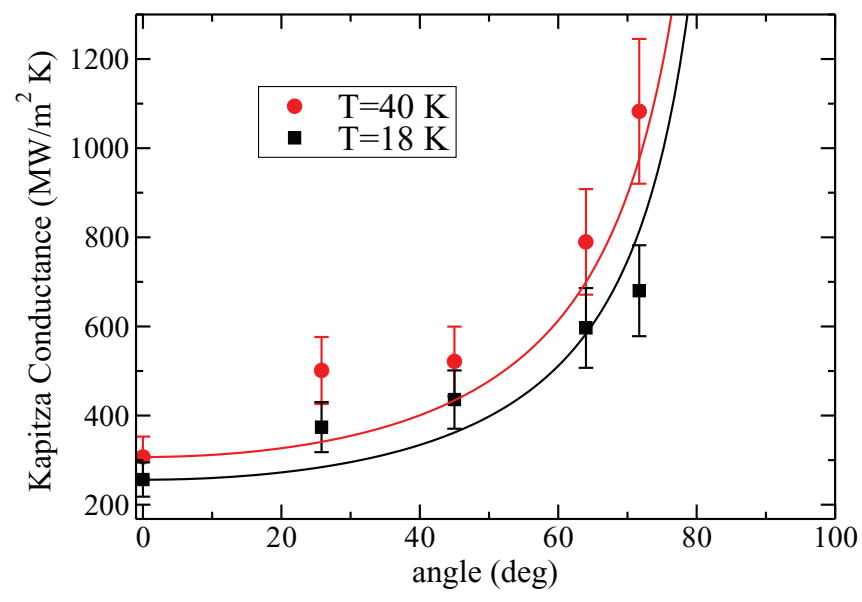

FIG. 8. (Color online) Same as Fig. 7, but for a constant value of the true surface area. The interfacial heights are, respectively, $h=21,34,43$, and 45 monolayers for the asperities angles $\alpha=$ $25.8,45,64$, and $71.7 \mathrm{deg}$. The solid lines show the interfacial conductance rescaled by the true surface area: $G_{K}=G_{K}(\alpha=0) / \cos \alpha$. 
conductance measured may even exceed the rescaled one. We have no interpretation for these large values reported here. Further increasing the angle $\alpha$, the simulation data take values close to the scaled conductances $G_{K}(\alpha=0) / \cos \alpha$. Note, in particular, that the increase of the conductance is pretty large, overpassing the conductance of a planar interface by a factor larger than three. In this regime, and for these steep interfaces, it is highly probable that the regime of rough interfaces, in terms of the previous discussion, has been reached: heat transmission becomes controlled by the true surface area. These large enhancements of the Kapitza conductance open the way to design interfaces with tailored interfacial energy transport properties.

\section{Effect of the shape of the interfaces}

We end the presentation of the results with a discussion of the effect of the shape of the interface on the boundary conductance. All of the previous discussion concentrated on a model of triangular interfaces, and it is worth asking how general are the conclusions drawn from the study of the particular type of surfaces. To appraise this question, we have considered different shapes of the interfaces, as depicted in Fig. 9. The common characteristic of these surfaces is the mean interfacial height, here fixed at a value $h=12$ MLs. Different morphologies have been designed, ranging from the random interface to the case of the squarelike surface and wavy interface obtained by a sinusoidal modulation of the interfacial height.

Figure 10 compares the interfacial conductance for the different shapes shown before, at two different temperatures. The relatively large values reported at the highest temperature may be explained by inelastic phonon scattering taking place between two interfaces. The shape of the interface seems to considerably affect interfacial transport: random interfaces display a conductance practically equal to the planar interface. Rough interfaces may transfer energy slightly better than planar interfaces depending on the temperature. On the other hand, wavy and squarelike interfaces tend to favor energy transmission, with the wavy pattern displaying the highest conductance among the different shapes analyzed. These results may be interpreted qualitatively: random- and rough-shaped interfaces display a distribution of length scales,

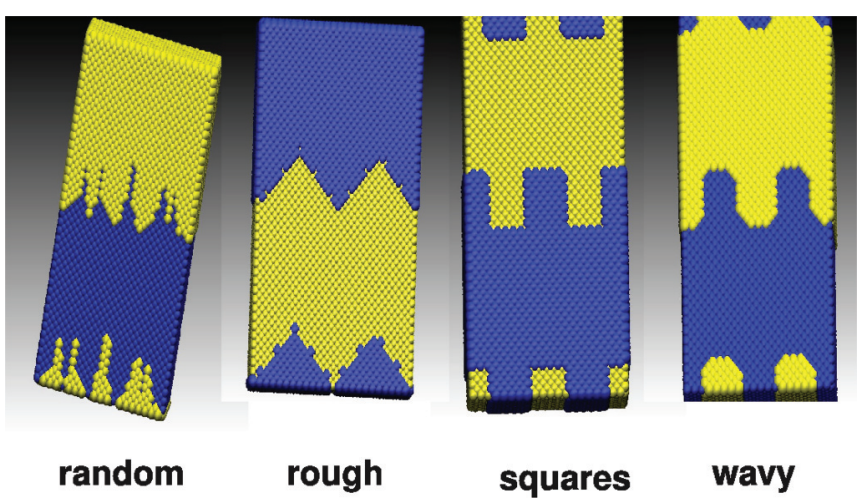

FIG. 9. (Color online) Illustration of the different interfacial shapes simulated, namely, random, rough, square, and wavelike interfaces.

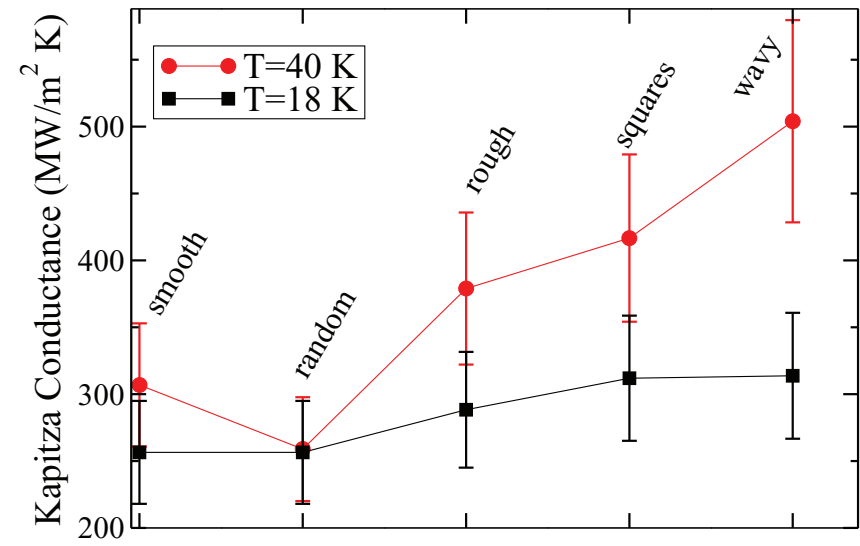

FIG. 10. (Color online) Thermal boundary conductance for the different interfacial shapes represented in Fig. 9. The height of the different interfaces is fixed here and equal to $12 \mathrm{ML}$. The other parameters are total length $=40 a_{0}$ and mass ratio $m_{r}=2$.

which tend to promote phonon scattering. Even if the global height $h$ is large, in terms of a triangular-shaped interface, the effective area for the phonons is not the true surface area, but rather the projected area, because $h$ is not the only relevant roughness parameter and the interaction between incident phonons and small length-scale asperities tend to diminish the effective transmission area. On the other hand, regular-shaped patterns do not display such a distribution of length scales, and interfacial heat transport becomes controlled by the true surface area: as soon as the majority of phonon modes has a wavelength greater than the single length $h$ characterizing the interfacial morphology, one enters into a "large roughness" regime, where the energy transport becomes governed by the true surface area and the conductance is increased as compared with the planar case. The interfacial conductance is found to be the highest for the wavy interface because it has the greatest surface area.

\section{CONCLUSION}

In summary, we have concentrated on the role of the interfacial roughness on energy transmission between solid dielectrics. Thanks to the versatility of the molecular dynamics simulations technique, we have conceived a model of rough surfaces and probe their ability to conduct heat. The scenario emerging from the simulations is the following: when the roughness introduced is small, most of the phonons see the interface as a planar one and the effective surface area contributing to the transmitted heat flux is the projected area, not the true one. In this regime, one does not expect a Kapitza conductance much different from the planar interface. On the other hand, when the roughness becomes large enough, typically 20 monolayers in our case, most of the phonons propagating towards the interface are incoherently scattered and the effective surface area becomes the true surface area. This latter case may differ significantly from the projected one, and this is the reason why the boundary conductance of rough interfaces may be greatly enhanced, as compared to planar interfaces. This has been demonstrated in this work with the example of triangular-shaped interfaces displaying 
steep slopes: provided the lateral dimensions characterizing the interfacial roughness are large enough, the increase of the conductance may be threefold. On the other hand, we have probed the conductance of randomly rough interfaces and shown that they display, in general, conductances comparable or smaller than atomic planar interfaces. This difference of behavior is explained by the distribution of length scales displayed by the randomly rough surfaces, in comparison with our model patterned surfaces.

The roughness analyzed in this paper was large compared to the lattice constants. The case of atomic roughness has been more widely addressed in the literature, and wave-packet simulations [36] give a clear picture of the effect of small atomic roughness on phonon transmission: long wavelength phonons see the interface as ideal and do not contribute to the change of the thermal boundary conductance. On the other hand, short wavelength phonons strongly interact with the small-scale roughness, and the corresponding change in phonon transmission is found to depend on the structure of the interface: for regular-shaped patterned interfaces, constructive wave interference leads to enhanced transmission, thereby increasing the boundary conductance [37]. Random atomic roughness promotes incoherent phonon scattering, reducing the thermal conductance. These observations are consistent with MD results concerning the cross-plane conductivity of superlattices with rough interfaces: for regularly patterned interfaces, the cross-plane conductivity is slightly greater than ideal superlattices [20] and the boundary conductance is enhanced [38]. When the roughness is random, the cross-plane conductivity shows a small decrease as compared with planar interfaces $[39,40]$. The small amplitude of the reduction is related to the small proportion of energy carriers affected by the atomic roughness. Small reductions have been also reported for $\mathrm{Si} / \mathrm{Ge}$ superlattices with one layer of interfacial mixing in the incoherent regime of transport [41]. If it is reasonable to rationalize such variations in terms of an atomic interfacial roughness, it is less clear for superlattices having a thicker mixed layer. Large enhancements have been observed in this latter case using MD [25]. Further work is clearly needed to understand if part of these enhancements is explained by the large-scale interfacial roughness [37].

Most of the results reported here concern regular-shaped patterned interfaces. MD results seem to conclude that these patterned interfaces are good candidates to enhance the intrinsic boundary conductance between two semiconductors. An enhancement by a factor of three has been reported for triangular-shaped interfaces; see Fig. 8. On the other hand, randomly rough surfaces should be considered if one prefers to reduce the Kapitza conductance between two solids [10]. In particular, in the context of superlattices, randomly rough interfaces should be designed if one aims to tailor materials with the lowest cross-plane thermal conductivity.

We have also introduced a simple model to rationalize the variations of the thermal boundary conductance as a function of the interfacial height of our model of rough interfaces. Further analytical work is clearly desired to understand the interplay between the interface morphology and energy interfacial transport. This will enable the definition of new directions for the design of interfaces with optimized energy transport properties and with a relative low cost.

\section{ACKNOWLEDGMENTS}

Simulations have been run at the "Pole Scientifique de Modélisation Numérique" de Lyon using the LAMMPS open source package [42]. We acknowledge interesting discussions with P. Chantrenne, T. Biben, P.-O. Chapuis, and D. Lacroix.
[1] H. Schneider, K. Fujiwara, H. T. Grahn, K. v Klitzing, and K. Ploog, Appl. Phys. Lett. 56, 6057 (1990).

[2] S. J. Wagner, J. Meier, A. A. Helmy, J. S. Aitchison, M. Sorel, and D. Hutchings, J. Opt. Soc. Am. B 24, 1557 (2007).

[3] J. L. Robert, F. Bosc, J. Sicart, and V. Mosser, Phys. Status Solidi B 211, 481 (1999).

[4] C. Wan, Y. Wang, N. Wang, W. Norimatsu, M. Kusunoki, and K. Koumoto, Sci. Technol. Adv. Mater. 11, 044306 (2010).

[5] A. Hashibon and C. Elsasser, Phys. Rev. B 84, 144117 (2011).

[6] B. Qiu, L. Sun, and X. Ruan, Phys. Rev. B 83, 035312 (2011).

[7] M. D. Losego, M. E. Grady, N. R. Sottos, D. G. Cahill, and P. V. Braun, Nat. Mater. 11, 502 (2012).

[8] P. J. O'Brien, S. Shenogin, J. X. Liu, P. K. Chow, D. Laurencin, P. H. Mutin, M. Yamaguchi, P. Keblinski, and G. Ramanath, Nat. Mater. 12, 118 (2013).

[9] B. Gotsmann and M. A. Lantz, Nat. Mat. 12, 59 (2013).

[10] P. E. Hopkins, L. M. Phinney, J. R. Serrano, and T. E. Beechem, Phys. Rev. B 82, 085307 (2010).

[11] P. E. Hopkins, J. C. Duda, C. W. Petz, and J. A. Floro, Phys. Rev. B 84, 035438 (2011).

[12] J. C. Duda and P. E. Hopkins, App. Phys. Lett. 100, 111602 (2012).
[13] D. Kekrachos, J. Phys. Condens. Matter 2, 2637 (1990).

[14] D. Kekrachos, J. Phys. Condens. Matter 3, 1443 (1991).

[15] J. Amrit, Phys. Rev. B 81, 054303 (2010).

[16] N. S. Shiren, Phys. Rev. Lett. 47, 1466 (1981).

[17] I. N. Adamenko and I. M. Fuks, Sov. Phys. JETP 32, 1123 (1971).

[18] Thermal Conductivity: Theory, Properties and Applications, edited by T. M. Tritt (Kluwer Academic/Plumer, New York, 2004).

[19] K. Termentzidis, J. Parasuraman, C. A. Da Cruz, S. Merabia, D. Angelescu, F. Marty, T. Bourouina, X. Kleber, P. Chantrenne, and P. Basset, Nano. Res. Lett. 6, 288 (2011).

[20] K. Termentzidis, S. Merabia, P. Chantrenne, and P. Keblinski, Int. J. Heat Mass Transfer 54, 2014 (2011).

[21] A. Rajabpour, S. M. W. Allaei, Y. Chalopin, F. Kowsary, and S. Volz, J. App. Phys. 110, 113529 (2011).

[22] K. Termentzidis, P. Chantrenne, and P. Keblinski, Phys. Rev. B 79, 214307 (2009)

[23] P. Chantrenne and J.-L. Barrat, J. Heat Transfer, Trans. ASME 126, 577 (2004).

[24] D. Frenkel and B. Smit, Understanding Molecular Simulation: From Algorithms to Applications (Academic, New York, 2002). 
[25] R. J. Stevens, L. V. Zhigilei, and P. M. Morris, Int. J. Heat Mass Transfer 50, 3977 (2007).

[26] E. S. Landry and A. J. H. McGaughey, Phys. Rev. B 80, 165304 (2009).

[27] S. Shenogin, L. Xue, R. Ozisik, P. Keblinskli, and D. G. Cahill, J. App. Phys. 95, 8136 (2004).

[28] L. Hu, T. Desai, and P. Keblinski, Phys. Rev. B 83, 195423 (2011).

[29] S. Merabia, P. Keblinski, L. Joly, L. J. Lewis, and J. L. Barrat, Phys. Rev. E 79, 021404 (2009).

[30] H. Zhao and J. B. Freund, J. App. Phys. 97, 024903 (2005).

[31] W. Zhang, T. S. Fisher, and N. Mingo, J. Heat Transfer 129, 483 (2007).

[32] P. E. Hopkins and P. M. Norris, ASME J. Heat Transfer 131, 022402 (2009).

[33] S. Merabia and K. Termentzidis, Phys. Rev. B 86, 094303 (2012).
[34] Periodic boundary conditions used in all directions impose the upper bound of the phonon wavelength.

[35] W. A. Little, Can. J. Phys. 37, 334 (1959).

[36] L. Sun and J. Y. Murthy, J. Heat Transfer 132, 102403 (2010).

[37] Z. Tian, K. Esfarjani, and G. Chen, Phys. Rev. B 86, 235304 (2012).

[38] X. W. Zhou, R. E. Jones, C. J. Kimmer, J. C. Duda, and P. E. Hopkins, Phys. Rev. B 87, 094303 (2013).

[39] B. C. Daly, H. J. Maris, K. Imamura, and S. Tamura, Phys. Rev. B 66, 024301 (2002).

[40] K. Termentzidis, P. Chantrenne, J.-Y. Duquesne, and A. Saci, J. Phys.: Condens. Matter 22, 475001 (2010).

[41] E. S. Landry and A. J. H. McGaughey, Phys. Rev. B 79, 075316 (2009).

[42] S. Plimpton, J. Comp. Phys. 117, 1 (1995); see http://lammps.sandia.gov. 